\title{
Secondary Cytoreduction Versus Chemotherapy Alone in the Treatment of Patients with Recurrent Ovarian Cancer: Is a Randomized Trial Worthwhile?
}

\author{
Paolo Sammartino • Daniele Biacchi • Marialuisa Framarino
}

Published online: 28 February 2013

(C) Springer Science+Business Media New York 2013

The review by Harter et al. [1] entitled "Surgery for relapsed ovarian cancer: when should it be offered?" appropriately analyzes major concerns related to surgery for recurrent ovarian cancer. Despite underlining published reports from various series on secondary cytoreduction in platinum-sensitive patients, seeking a satisfactory answer to the question what should be considered standard care in these patients, they present two ongoing randomized trials (AGO-OVAR DESKTOP III and GOG 213) evaluating cytoreductive surgery versus chemotherapy alone in patients with platinum-sensitive recurrent ovarian cancer.

Even though we agree that these studies are needed to obtain level 1 evidence for choosing the optimal treatment in similar clinical scenarios, we feel obliged to express and draw to the scientific community's attention certain doubts. From currently available data, no one can deny that in selected cases such as the categories identified by the inclusion criteria for the AGO-OVAR DESKTOP III trial, the results obtained after secondary surgical cytoreduction in patients with recurrent ovarian cancer are better than those after chemotherapy alone $[2,3]$. This consideration raises ethical and practical concerns. As physicians engaged in scientific research, it is our duty to suggest to our patients the therapeutic option likely to guarantee the best outcome. Equally important, when asking patients to take part in a randomized trial, we have the duty to inform them about the reasons for the trial and the results that

\section{P. Sammartino $(\bowtie) \cdot$ D. Biacchi}

Department of Surgery "Pietro Valdoni",

Sapienza University of Rome, Azienda Policlinico Umberto I,

Viale del Policlinico 155

00161 Rome, Italy

e-mail: paolo.sammartino@uniroma1.it

\section{Framarino}

Department of Gynecology and Obstetrics,

Sapienza University of Rome, Rome, Italy the two alternative procedures proposed can guarantee. Giving correct information could presumably lead to an accrual dropout.

No less important in this context are the latest developments from cytogenetic studies on ovarian carcinoma. In their critical review published on the pathogenesis of ovarian cancer, Kurman and Shih [4] divide epithelial ovarian carcinoma into two groups designated type I and type II that distinctly differ in phenotype, genotype, and clinical behavior. These new concepts on ovarian carcinogenesis inescapably imply that a "blanket approach" to the treatment of ovarian carcinoma no longer suffices. The overriding need now is to develop targeted therapies adapted to the molecular and genetic features shown by the two main tumor types. Randomized trials that fail to take these differences into account can hardly provide reliable results.

Conflict of Interest Paolo Sammartino declares no conflict of interest.

Daniele Biacchi declares no conflict of interest.

Marialuisa Framarino declares no conflict of interest.

\section{References}

1. Harter P, Heitz F, du Bois A. Surgery for relapsed ovarian cancer: when should it be offered? Curr Oncol Rep. 2012;14(6):539-43.

2. Zang RY, Harter P, Chi DS, et al. Predictors of survival in patients with recurrent ovarian cancer undergoing secondary cytoreductive surgery based on the pooled analysis of an international collaborative cohort. Br J Cancer. 2011;105(7):890-6.

3. Ledermann JA, Raja FA. Clinical trials and decision-making strategies for optimal treatment of relapsed ovarian cancer. Eur J Cancer. 2011;47 Suppl 3:S104-15.

4. Kurman RJ, Shih I-M. Pathogenesis of ovarian cancer: lessons from morphology and molecular biology and their clinical implications. Int J Gynecol Pathol. 2008;27(2):151-60. 